\title{
djb-Stellungnahme zur Reform der Strafvorschriften des Menschenhandels, Verbesserung des Schutzes der Opfer von Menschenhandel und Regulierung der Prostitution'
}

\author{
Ramona Pisal, \\ Präsidentin
}

Dagmar Freudenberg,

Vorsitzende der Kommission Strafrecht

\author{
Sabine Overkämping, \\ Vorsitzende der Kommission Öffentliches Recht, Europa- und \\ Völkerrecht
}

\section{Dr. Maria Wersig,}

Vorsitzende der Kommission Recht der sozialen Sicherung, Familienlastenausgleich

Menschenhandel und Prostitution sind sehr umstrittene Themen, die die Bundesregierung im Zuge der Bundestagswahl und der dann folgenden Koalitionsvereinbarung auf die politische Agenda gesetzt hat. Sie sind franenrelevant, so dass wir uns als djb damit auseinandersetzen müssen, zumal wir spätestens seit der Initiative von EMMA letztes Jahr immer wieder auf unsere rechtspolitische Meinung hierzu angesprochen wurden und werden. Die Ministerien wollten auf unsere Expertise bei der Suche nach sinnvollen Regelungen nicht verzichten und haben uns damit zeitlich ebenfalls sebr unter Druck gesetzt. Eine djb-Veranstaltung zu diesem Thema wäre sinnvoll (gewesen) und wir haben im Bundesvorstand darüber diskutiert, doch ein Seminar im Herbst 2014 oder die Mitgliederversammlung im Rahmen des Bundeskongresses im September 2015 in Münster konnten wir nicht abwarten.

Der Bundesvorstand musste daher tätig werden und hat in seiner Sitzung am 30. November 2013 beschlossen, einen Arbeitsstab unter der Verantwortung dreier Kommissionen (Strafrecht, Recht der Sozialen Sicherung/Familienlastenausgleich, Öffentliches Recht/Europa- und Völkerrecht) einzusetzen. Im Mitglieder-Newsletter vom 16. Dezember 2013 haben wir insbesondere Fachfrauen im Gewerbe- und Gesundheitsrecht um Bewerbungen zur Mitarbeit gebeten. 2014 fanden zwei Sitzungen und umfangreiche Abstimmungen per E-Mail mit insgesamt 14 djb-Mitgliedern (Vertreterinnen der drei djb-Kommissionen und weiteren djb-Fachfrauen) statt. Die Arbeiten waren intensiv und aufwändig, u.a. gab es auch einen Expertinnenworkshop mit Vertreterinnen der Prostituierten und der Beratungsstellen der Opfer von Menschenhandel. Zu einigen besonders umstrittenen Punkten konnte kein Konsens erzielt werden, sondern es wurden Mehrheitsentscheidungen getroffen. Das haben wir in der Stellungnabme vom 15. September 2014 deutlich gemacht. Alle Kolleginnen, die sich in der einen oder anderen Richtung in die Stellungnahme eingebracht haben, haben ernsthaft um eine mit ihrem Gewissen vereinbare Lösung gerungen. Das zeigt, dass der djb ein lebendiger Expertinnen-Verein ist, der nicht ausgrenzt, sondern sich argumentativ auseinandersetzt. Wir geben nunmehr allen djb-Mitglieden die Möglichkeit, sich hierzu zu äußern. Ibre Kommentare werden in der djbZ 1/2015 und/oder 2/2015 abgedruckt (max. 2.000 Zeichen, damit wir viele Perspektiven abdrucken können; Kürzungen behalten wir uns vor). Wir freuen uns auf ein breites Meinungsbild.

Der Deutsche Juristinnenbund e.V. (djb) hat zur Frage der Umsetzung der Richtlinie 2011/36/EU und zur Diskussion des weiteren rechtspolitischen Handlungsbedarfs zur Bekämpfung des Menschenhandels, Unterstützung der Opfer von Menschenhandel, Stärkung der Prävention und Regulierung des Prostitutionsgewerbes einen Arbeitsstab eingesetzt, der von Februar bis August 2014 gearbeitet und sich mit aktuellen Forderungen zu Reformen in den genannten Bereichen auseinandergesetzt hat. Der Arbeitsstab hat darüber hinaus eine Anhörung durchgeführt, bei der Vertreterinnen von Beratungsstellen, die mit Opfern von Menschenhandel arbeiten, sowie Vertreterinnen der Verbände von Sexarbeiterinnen anwesend waren. Die Ergebnisse und Empfehlungen aus der Arbeit des Arbeitsstabs fasst diese Stellungnahme zunächst zusammen, das ausführliche Gutachten folgt im Anschluss.

Zusammenfassend empfiehlt der djb:

1. Maßnahmen zur Bekämpfung des Menschenhandels, Stärkung des Opferschutzes und der Prävention (Umsetzung der EU-Richtlinie 2011/36/EU):

Die Richtlinie 2011/36/EU legt Mindestvorschriften zur Definition von Straftaten und Strafen im Bereich des Menschenhandels, Bestimmungen zur Stärkung des Opferschutzes und zur Stärkung der Prävention fest. Die Frist zur Umsetzung dieser Richtlinie endete am 6. April 2013. Deutschland droht wegen Nichtumsetzung ein Vertragsverletzungsverfahren. Der bestehende Umsetzungsbedarf sollte für eine umfassende Reform genutzt werden. Der djb empfiehlt:

- Die Neufassung des Straftatbestands des Menschenhandels im Strafgesetzbuch.

- Die Verbesserung der strafprozessualen Bestimmungen zur Stärkung des Opferschutzes.

- Die Begründung von Aufenthaltsregelungen für Opfer von Menschenhandel, die unabhängig sind von der Bereitschaft zur Kooperation im Strafverfahren. Die vom Referentenentwurf

1 Bei der abgedruckten Stellungnahme handelt es sich um eine kurze Zusammenfassung. Die Langversion finden Sie online auf der djb-Webseite: http://www.djb.de/st-pm/st/st14-16/Langfassung/ (Zugriff: 27.10.2014) 
des Bundesministeriums des Inneren (BMI)2 vorgeschlagene Ergänzung des $\$ 25$ Abs. 4a AufenthG erreicht die notwendige Entkoppelung von Aussagebereitschaft bzw. Zeugenaussage von der Gewährung des Aufenthaltsrechts nicht.

- Der Schutz der Opfer von Menschenhandel ist in der Praxis zu verbessern. Wichtig ist dabei vor allem die Kooperation von Fachberatungsstellen und der Polizei. Der djb empfiehlt eine bundesweite Evaluation aller bestehenden Kooperationskonzepte und ihrer Umsetzung. Ferner wird empfohlen, bei allen Polizeidienststellen bundesweit Fachkommissariate für Menschenhandel einzurichten.

- Die Verfolgung von Mietwucher sollte im Rahmen der Bekämpfung des Menschenhandels intensiviert werden.

- Vermögenssichernde Maßnahmen (Beschlagnahme, Gewinnabschöpfung) finden in Strafverfahren wegen Menschenhandels aktuell nur in erstaunlich geringem Umfang statt. Diese aktuelle Praxis stellt eines der größten Hindernisse bei der Durchsetzung von Schadensersatzansprüchen von Betroffenen dar. Ziel der Praxis muss es sein, die erwirtschafteten Gewinne effektiv zu entziehen. Es gilt, die vorhandenen rechtlichen Möglichkeiten auszuschöpfen.

- Der djb spricht sich gegen ein Verbot der Prostitution und gegen eine allgemeine Strafbarkeit der Freier aus.

- Ein Verbot der Prostitution würde diese insgesamt in die Illegalität zurücktreiben, den Menschenhandel jedoch nicht verhindern, sondern lediglich seine Aufdeckung noch weiter erschweren. Ein Verbot der Prostitution wäre grundgesetzlich wegen des Eingriffs in Art. 12 GG nur dann zu rechtfertigen, wenn jedwede Form der Ausübung dieses Berufs als menschenrechtswidrig und der Gesellschaft schädlich eingestuft werden müsste. Dies ist nach der überwiegenden Auffassung im Arbeitsstab und ausweislich einer Mitgliederbefragung - unter den Mitgliedern des djb nicht der Fall. ${ }^{3}$ Vielmehr gilt es, Abhängigkeits- und Ausbeutungsstrukturen zu identifizieren und zu bekämpfen. Das wird nicht durch ein Verbot erreicht werden können, sondern durch gezielte Regulierung des Prostitutionsgewerbes.

- Eine generelle Strafbarkeit der Freier, wie sie z.B. in Schweden erfolgt ist, würde den Kernbereich von Art. 12 GG verletzen. Eine Strafbarkeit der Freier, die Dienste von Menschenhandelsopfern unter Kenntnis deren Situation in Anspruch nehmen, könnte eine erlaubte Bekämpfung strafwürdiger Verhaltensweisen darstellen. Es ist allerdings zweifelhaft, ob dadurch die Bekämpfung des Menschenhandels effektiviert werden könnte. Freier, die den Strafverfolgungsbehörden mögliche Opfer von Menschenhandel benennen, würden sich gleichzeitig der Gefahr einer Bestrafung aussetzen. Ein wichtiges Beweismittel, die Zeugenaussage von Freiern, könnte somit verlorengehen. Diese Folge könnte allenfalls durch die gleichzeitige Einführung einer Vorschrift der fakultativen Straflosigkeit aufgrund tätiger Reue vermieden werden.

\section{Zum Thema Regulierung des Prostitutionsgewerbes:}

Eine Kriminalisierung der Prostitution oder der Inanspruchnahme sexueller Dienstleistungen mit dem Ziel der Bekämpfung des Menschenhandels ist aus Sicht des djb wenig erfolgversprechend.
Sie stünde außerdem im Widerspruch zu der vom Bundesverfassungsgericht ausdrücklich vorgenommenen Zuordnung der Prostitution zu Art. 12 GG und würde die Situation der aus eigener freier Entscheidung in der Prostitution tätigen Frauen (und Männer) verschlechtern. Vielmehr sollte eine weitere Regulierung des Prostitutionsgewerbes erfolgen, mit dem Ziel der Etablierung einheitlicher und transparenter Anforderungen an Betreibende von Prostitutionsstätten, Standards für gute Arbeitsbedingungen und den Schutz der sexuellen Selbstbestimmung der im Prostitutionsgewerbe tätigen Frauen (und Männer). Insofern sind die im Eckpunktepapier „Eckpunkte eines Gesetzes zum Schutz der in der Prostitution Tätigen (Prostituiertenschutzgesetz, ProstSchG) “ vom 14. August 2014 des Bundesministeriums für Familie, Senioren, Frauen und Jugend (BMFSFJ) benannten Ziele der Stärkung des Selbstbestimmungsrechts von Prostituierten (männlich/weiblich) und der Gewährleistung verträglicher Arbeitsbedingungen und des Gesundheitsschutzes zu begrüßen. Aufsuchende Beratungs- und Informationsangebote sowie auf freiwillige anonyme Inanspruchnahme gerichtete Angebote der Gesundheitsversorgung sollten außerdem verbessert werden. Des Weiteren empfiehlt der djb:

- Die gewerberechtliche Regulierung des Prostitutionsgewerbes in einem Prostitutionsstättengesetz, das Anforderungen für den Betrieb von Prostitutionsstätten aufstellt. Dabei soll leitendes Ziel die Sicherstellung und Kontrolle guter Arbeitsbedingungen und Sicherung der sexuellen Selbstbestimmung von Prostituierten sein. Eine Erlaubnispflicht für das Betreiben einer Prostitutionsstätte, wie das Eckpunktepapier des BMFSFJ vorschlägt, unterstützt der djb ausdrücklich.

- Eine individuelle Anzeige-/Anmeldepflicht, wie sie das Eckpunktepapier des BMFSFJ für Prostituierte in den Raum stellt, lehnt der djb ab, weil die Gefahr der Stigmatisierung evident ist und hohe Anforderungen an die Erhebung und Verarbeitung von Daten, die mit der Sexualität eines Menschen zu tun haben, im Europarecht und bundesdeutschem Recht bestehen. Auch eine Pflicht zur Offenlegung der Namen gegenüber Kunden durch Bereitstellung eines „Nachweisdokuments“ könnte Gefahren und Persönlichkeitsrechtsverletzungen zur Folge haben.

- Verpflichtende regelmäßige Gesundheitsuntersuchungen für die Gruppe der Prostituierten sind verfassungsrechtlich problematisch und faktisch kontraproduktiv, weil sie den Erfahrungen mit bewährten Strategien bei der Bekämpfung sexuell übertragbarer Krankheiten diametral widersprechen.

- Eine bundesweite Kondompflicht ist kaum durchsetzbar und birgt ebenfalls die Gefahr, dass Kontrollen und Sanktionen sich vor allem auf Prostituierte (und nicht ihre Kunden) negativ auswirken.

2 „Entwurf eines Gesetzes zur Neubestimmung des Bleiberechts und der Aufenthaltsbeendigung", Stand: 7.4.2014.

3 Der djb hat im Jahr 2000 eine Mitgliederbefragung zur Beurteilung der Prostitution als sittenwidrig durchgeführt. 89 Prozent der befragten Mitglieder hielten die Prostitution nicht für sittenwidrig und unterstützten demnach das wesentliche Ziel des Prostitutionsgesetzes von 2001. Online: <http://www.djb.de/Kom/ K3/pm-58/> (Zugriff: 11.9.2014). 